\title{
Analysis of LEA protein family members in Lepidium apetalum seeds and the expression of $L a L E A 1$ in seedlings in response to abiotic stresses
}

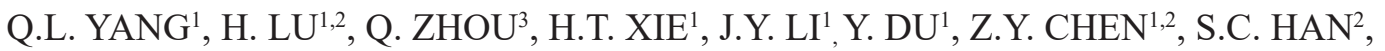 \\ H.P. ZHAO ${ }^{1,2}$, and H.X. ZHAO ${ }^{1 *}$ \\ College of Life Science, Xinjiang Normal University, Xinjiang Urumqi 830054, P.R. China ${ }^{1}$ \\ College of Life Sciences, Beijing Normal University, Beijing 100875, P.R. China ${ }^{2}$ \\ College of Life and Geographic Sciences, Kashgar University, Kashgar 8440001, P.R. China ${ }^{3}$
}

\begin{abstract}
To study the biological function of late embryogenesis abundant (LEA) proteins in Lepidium apetalum Willd., genes encoding LEA family proteins were identified from the seed transcriptome. The sequence most closely related to germination at a low temperature was selected and gene expressions in response to low temperature stress further studied. The results show that $27 L E A$ genes were expressed in seeds germinating at the low temperature: 3 genes were upregulated, 20 were downregulated, and 4 were not significantly different from controls. The most prominent of the upregulated genes, LaLEA1, contained an open reading frame of $624 \mathrm{bp}$ and encoded 208 amino acids. The protein was rich in hydrophilic amino acids including threonine, alanine, glutamine, and lysine. It is predicted that the secondary structure contains $\alpha$-helices and irregular curls. Real-time quantitative PCR results show that under low temperature stress, the expression of $L a L E A 1$ was first downregulated and then upregulated rapidly, reaching its highest expression at $12 \mathrm{~h}$, then the expression of $L a L E A 1$ was reduced slightly but maintained higher than that of the non-stress group. As the expression of LaLEA1 was significantly altered in response to low temperature stress, we investigated the expression of LaLEA1 also in response to other abiotic stresses, i.e., salinity and drought. L. apetalum seedlings wilted in the early stage following $\mathrm{NaCl}$ or osmotic (polyethylene glycol) stresses, but recovered quickly, showing a strong tolerance. Real-time quantitative PCR results show that LaLEA1 was rapidly upregulated following salt and osmotic stresses, and its expression profile was closely related to $\mathrm{NaCl}$ or PEG concentrations. Expression was up to 7.9-fold higher than that of the control after $6 \mathrm{~h}$ of salt stress. These results suggest that $L$. apetalum seedlings responded quickly to salt stress. The response to osmotic stress was slightly slower; expression of LaLEA1 was 6.0-fold higher than that of the control after $12 \mathrm{~h}$. Thus, LaLEA1 played an important role in abiotic stress tolerance. These results provide a basis for further analysis of the role of the $L E A$ genes in the stress resistance of L. apetalum.
\end{abstract}

Additional key words: gene expression, low temperature, osmotic stress, salinity.

\section{Introduction}

Lepidium apetalum Willd. (family Brassicaceae), which has medicinal and nutritional value, is distributed worldwide from 400 to $2000 \mathrm{~m}$ above sea level. This plant has the ability to adapt to a widely differing environments producing different ecotypes. For example, the ecotype in northern Xinjiang (China) has been reported to grow at 0 to $5^{\circ} \mathrm{C}$ in some cases (Mao and Zhang, 1994). The climate in the mountainous areas of northern Xinjiang is very dry with some snow in winter. In early spring, ephemeral plants germinate and grow rapidly in the cold soil, which is soaked with melted snow, completing their life cycles before the soil dries up. This harsh environment makes L. apetalum living in northern Xinjiang, China unique in its ability to tolerate cold early spring weather, and to germinate and grow under such conditions (Yang et al. 2015). In a study of seed germination characteristics, it was found that the low-temperature germination of L. apetalum proceeds as follows: at temperatures of 0 to

Submitted 27 September 2019, last revision 15 December 2019, accepted 19 December 2019.

Abbreviations: LEA - late embryogenesis abundant; PEG - polyethylene glycol.

Acknowledgments: This work was supported by grants from the "13th Five-Year" Plan for Key Discipline Biology, Xinjiang Normal University (17SDKD0201), the National Natural Science Foundation of China (31660079 and 31460041), and the Open projects of the Beijing Key Laboratory of Resistance Genes Resource and Molecular Development (2017GD03), Research and Innovation Project for Graduate Students in Xinjiang Uygur Autonomous Region. The first two authors contributed equally to this work.

* Corresponding author; e-mail: 954843437@qq.com 
$5{ }^{\circ} \mathrm{C}$, the initial germination of seeds can occur, but there is a stagnation period. Interestingly, brief exposure (50 min) to a higher temperature $\left(\right.$ e.g. $\left.25^{\circ} \mathrm{C}\right)$ allows the seeds to circumvent the stagnation period. When the germinated seeds are again placed in a low-temperature environment, they can tolerate the low temperature, and continue to germinate and grow (Zhao et al. 2010). Considering the seed response to temperature, the transcriptome of seeds under stagnant, low-temperature conditions was compared to that of seeds resuming germination following a short, high-temperature treatment; differentially expressed genes were identified (Shah et al. 2018, Young et al. 2018). In addition to low-temperature damage, the plants also experience drought and salt stress during seed germination and seedling growth. Considering the harsh growth environment of L. apetalum, late embryogenesis abundant (LEA) proteins have recently been investigated; LEA family proteins play an important role in abiotic stress tolerance in plants, including cold, drought, and salt stresses (Xiang et al. 2018).

LEA proteins are a class of small hydrophilic proteins that were first isolated from cotton embryos by Capdevila and Dure (1981). These proteins are widespread throughout eukaryotic organisms, including plants (Liang et al. 2016, Yu et al. 2016, 2017, Lin et al. 2017, He et al. 2018). According to homologous and conserved domains, the LEA protein family was divided into the groups D-19, D-11, D-7, D-113, D-29, D-34, and D-73, as well as several additional groups. Their common characteristic is high content of glycine (Gly), lysine (Lys), arginine (Arg), glutamic acid (Glu), and threonine (Thr) (Arroyo et al. 2000). LEA proteins are highly hydrophilic and exhibit thermal stability. LEA proteins have been reported to be involved in regulating the embryonic development (Galau et al. 1987, Ingram and Bartels 1996, Oliveira et al. 2010). There is also evidence that plant LEA proteins play a role in plant tolerance to abiotic stresses (Cai et al. 2006, Wang et al. 2008, 2011, Jiang et al. 2017). LEA proteins are thought to regulate plant stress resistance by stabilizing proteins, thus protecting against denaturation (Liu, 2014, $\mathrm{He}$ and $\mathrm{Hu} 2015$, Hu et al. 2015), reactive oxygen species (Liu, 2011, Li et al. 2017a), or by stabilizing the cell membrane (Mouillon et al. 2006, Alan and Wise 2007). However, LEA family proteins differ significantly among different plants, and the mechanism underlying the regulation of plant development and stress resistance by LEA proteins is not fully understood.

The seedlings of $L$. apetalum also exhibited strong tolerance to low temperature (Meng et al. 2008, Zhao et al. 2010, Yang et al. 2015, Li et al. 2016, 2017b, Yuan et al. 2018, Zhao et al. 2018). The transcriptomes of the two different groups of seeds were sequenced (Zhou et al. 2016) and many highly expressed LEA protein family genes were identified. However, whether the expressions of these genes were related to tolerance of the seed germinated at low temperature, or to the other abiotic stresses, has not yet been determined. In this study, expressions of LEA genes in the two groups were analyzed, before and after lowtemperature germination. Expression of the LaLEA1 gene under salinity and drought was also investigated. These results provide an experimental basis to better understand the function of $L E A$ genes and the adaptation mechanisms of L. apetalum seedlings.

\section{Materials and methods}

Plant, treatments, and related data sources: Lepidium apetalum Willd. ripe seeds were collected from Liyushan mountain, Urumqi, Xinjiang in northern China. After drying at room temperature, the seeds were stored at $4{ }^{\circ} \mathrm{C}$. Seeds were treated with $98 \%(\mathrm{~m} / \mathrm{v})$ sulfuric acid to remove the seed coat, rinsed with distilled water several times, placed on wet filter paper, and left at a temperature of $24-26^{\circ} \mathrm{C}$, a $16-\mathrm{h} \cdot$ photoperiod, an irradiance of 400 to $650 \mu \mathrm{mol} \mathrm{m} \mathrm{m}^{-2} \mathrm{~s}^{-1}$, and a relative humidity of $60 \pm 5 \%$ to germinate for 1 week. Seven-day-old seedlings of uniform size were selected to clone the cDNA of the LaLEA1 gene, and to verify the response of the LaLEA1 gene to abiotic stress. LEA protein information of the seeds of $L$. apetalum was obtained from our previous transcriptome sequencing results. Gene sequence information for other species was obtained from the $N C B I$ database.

Screening for LEA protein family genes: The seeds which had been deposited at $4{ }^{\circ} \mathrm{C}$ for $9 \mathrm{~d}$ were used as the sample of germination at low temperature. After that some seeds were exposed to $25^{\circ} \mathrm{C}$ for $55 \mathrm{~min}$. Sequencing libraries were constructed for the both seed groups (Zhou et al. 2016, 2018).

High throughput sequencing using Illumina HiSeqTM2000 was then performed. Using LEA as the key word, $L E A$ gene related sequences were obtained from the transcriptome data of $L$. apetalum seeds. Each sequence screened from the transcriptome database was analyzed in the NCBI database for BLAST analysis (https:// www. ncbi.nlm.nih.gov). Only those sequences that contain the conservative domain of $L E A$ and are highly homologous (consistency greater than $85 \%$ ) with the sequences annotated as $L E A$ in $N C B I$ can be identified as members of the $L E A$-related family. Following removal of redundant sequences, cDNA sequences of $L E A$ family members of L. apetalum were determined. Functional determination of LEA protein family genes was carried out using the nonredundant $(\mathrm{Nr})$ protein and eukaryotic ortholog groups $(K O G)$ databases, as well as gene ontology $(G O)$ analysis.

Extraction of total RNA from seedlings of Lepidium apetalum: TRIzol reagent was used to extract total RNA from ??-d-old seedlings. RNA purity and quality were detected by agarose gel electrophoresis and a UV spectrophotometer. The cDNA synthesis was carried out using a reverse transcription kit (RevertAid First Strand cDNA synthesis kit) according to the manufacturer's instructions. The cDNAs were stored at $-80{ }^{\circ} \mathrm{C}$ until further use.

Differential expression analysis of LEA protein family genes before and after low-temperature germination: Based on the fragments per kilobase of transcript per 
million fragments mapped (FPKM) method, differential expression analysis of LEA protein coding genes in the transcriptome was performed. Genes that were up- or down-regulated more than 0.5-1.5 times were considered differentially expressed. Target genes of $L$. apetalum seeds with significant differences in expression before versus after germination at low temperature were identified.

Cloning and sequence analysis of the fulllength cDNA sequence of LaLEA1: Based on the transcriptome sequencing results of the seeds, specific primers for the amplification of LaLEA1 (LaLEA1-2F, 5'-CGGGGTACCAACAAAAATGGCGTCTCACC-3' and LaLEA1-2R, 5'-ACGCGTCGACGCTCACTTCCTC TGATA-3') were designed. Using the cDNA of L. apetalum seedlings as a template, the full-length sequence of the LaLEA1 gene was amplified (commissioned by the Beijing PMAD company for sequencing). DNAMAN software was used to compare these sequences with the transcriptome sequences. The NCBI database was used to analyze sequence homology. The properties of the protein were analyzed by ProtParam and the secondary structure was predicted using ExPASy. SWISS-MODEL was used to predict the tertiary structure of the proteins. By using $M E G A 6$ software to align amino acid sequences, and based on the maximum likelihood method, the phylogenetic tree was constructed through 500 bootstrap tests.

Design of real-time fluorescent quantitative PCR primers to assess $L \boldsymbol{L} L \boldsymbol{E} \boldsymbol{A} 1$ expression: According to the transcriptome sequencing results of $L$. apetalum seeds and the obtained sequence of $L a L E A 1$, the following fluorescent quantitative PCR primers were designed: LaLEA1Rq-2F, 5'- AGACAAAGCTCGTGAGTCCA -3' and LaRqLEA2R, 5'-TTCGTCCTCTGTAGCCATCC-3'. $\beta$-actin was used as an internal reference gene. The specific primers P1, 5'-CCAAAGGCCAACAGAGAGAAGAT-3' and P2, 5'-TGAGACACACCATCACCAGAAT-3' were also used.

Expression of $\mathrm{LaLEA1}$ in response to abiotic stresses: For low temperature stress, 7-d-old seedlings were placed in a $0{ }^{\circ} \mathrm{C}$ for $0,1,2,4,8,12,24$, and $48 \mathrm{~h}$. For salt stress, 7-d-old seedlings were cultivated in media containing different $\mathrm{NaCl}$ concentrations (50, 100, 150, 200, 250, and $300 \mathrm{mM}$ ). For osmotic stress, 7-d-old seedlings were cultivated in media with $10,15,20,25$, or $30 \%(\mathrm{~m} / \mathrm{v})$ polyethylene glycol (PEG 6000). The last two treatments were performed for $0,1,6,12,24,36$, and $48 \mathrm{~h}$. The LaLEA1 gene expressions in response to cold, salt, and osmotic stresses were analyzed using a fluorescence quantitative method. The seedlings grown in media without additions and at room temperature were used as control plants.

Statistical analysis: The Student's $t$-test was used for analysis of statistical significance following a Gaussian distribution. Results are expressed as means \pm standard errors (SEs), $n=3$. Different letters (a-e) indicate significant differences $(P<0.05)$.

\section{Results}

Based on the transcriptome sequencing results of L. apetalum seeds, 35 sequences containing the key word LEA were identified. Following removal of redundant sequences, 27 LEA gene sequences were identified. The phylogenetic tree was constructed using 27 LEA protein enconding gene sequences, which showed that all of them belong to the LEA protein family (Fig. 1 Suppl.). The longest and shortest sequences were 882 and $164 \mathrm{bp}$, respectively. Of the 27 LEA sequences, seven encoded complete open reading frames.

Lepidium apetalum late embryogenesis abundant (LEA) protein family unigene distribution based on the $\mathrm{Nr}$ database and $G O$ analysis indicated that the identified $L E A$ family genes were highly homologous to known $L E A$ genes of five species of Brassicaceae, $63 \%$ were homologous with those of Arabidopsis thaliana (Fig. 1).

The predicted functions of the products encoded by the $27 L E A$ genes of $L$. apetalum were obtained by $G O$ analysis. In total, $21 \mathrm{LEA}$ genes were annotated to the biological process category, 10 to the molecular function category, and 15 to the cellular component category (Fig. 2). Among the 27 LEA genes, only 8 were annotated to the three categories. It is speculated that these 8 genes may have multiple functions in plant development and stress resistance (Fig. 2 Suppl.). Three genes were not annotated to terms in the GO database.

Based on the conserved sites, the plant LEA protein sequences were grouped into 26 functional components

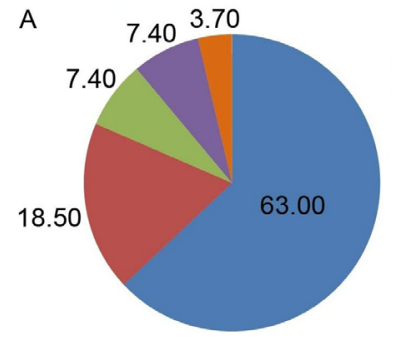

$[\%]$

- Arabidopsis thaliana

- Arabidopsis lyrata subsp. Lyrata

- Capsella rubella

- Brassica napus

- Eutrema salsugineum

B biological process

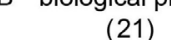


using the $K O G$ database. In this study, 5 LEA protein family gene sequences isolated from the seeds of $L$. apetalum were functionally annotated in $K O G$. Only one sequence was reported, which was annotated as $\mathrm{O}$ (post-translational modification, protein turnover, chaperones); the remaining four sequences were annotated as $\mathrm{S}$ (function unknown) (Table 1). The other 22 sequences were not annotated in the KOG database.

LEA proteins generally contain a highly conserved motif rich in alkaline and hydrophilic amino acids, and their structures are primarily composed of $\alpha$-helices. LEA proteins are highly hydrophilic, and when subjected to abiotic stresses such as drought, salinity, and high temperature, are able to maintain function of membrane.
The 27 LEA protein family genes expressed during seed germination belonged to the groups D-7, D-113, D-73, and LEA_2 based on analysis of conserved domains. The hydrophilic features of these proteins resulted in their high thermal stability (Table 1 Suppl.).

The transcriptional data of LEA protein family genes of $L$. apetalum seeds were analyzed before and after germination at low temperature (Fig. 2). We conducted RT-qPCR to validate the RNA-seq data and to analyze the gene expression changes of randomly selected genes. Although the expressions of selected genes were different between RNA-seq and RT-qPCR, the trend of expressions were the same (Fig. 3 Suppl.). Three upregulated and twenty downregulated genes were identified, the

Table 1. Karyotic ortholog group classification of late embryogenesis abundant protein family genes of Lepidium apetalum.

\begin{tabular}{llll}
\hline Transcript sequence & KOG ID & Acronym for KOG class & Description \\
\hline c37384_g1 & KOG4744 & S & unknown function \\
c34997_g1 & KOG4744 & S & unknown function \\
c18705_g1 & KOG4098 & O & posttranlation modification, protein turnover, chaperones \\
c15290_g1 & KOG4744 & S & unknown function \\
c23918_g1 & SKOG4744 & & unknown function \\
\hline
\end{tabular}

\begin{tabular}{|c|c|c|c|c|c|}
\hline GROUP & SERIAL NUMBER & LENG & HOMOLOGLUS GENES & CONTROL MEAN & EXPERIMENTAL MEAN \\
\hline \multirow{4}{*}{ Group 2} & c12286_g1 & 565 & Arabidopsis thaliana dehydrin & & \\
\hline & c10995_g1 & 405 & Arabidopsis thaliana dehydrin & & \\
\hline & c36763_g1 & 114 & A rabidopsis lyrata subsp. lurata ECP 63 & & \\
\hline & c23918_g1 & 555 & Arabidopsis thaliana $L E A 7$ & & \\
\hline \multirow[t]{4}{*}{ Group 3} & c15290_g1 & 882 & Arabidopsis lyrata subsp. Iyrata LEA 1 & & \\
\hline & c37384_g1 & 693 & Camelina sativa $L E A 76$ & & \\
\hline & c10628_g1 & 228 & A rabidopsis thaliana $L E A$ & & \\
\hline & c35254_g1 & 411 & A rabidopsis thaliana LEA 4-1 & & \\
\hline \multirow{3}{*}{ Group 4} & c8434_g1 & 522 & Thellungiella salsuginea LEA 1 & & \\
\hline & c23990_g1 & 363 & Arabidopsis thaliana LEA 18 & & \\
\hline & c34997_g1 & 750 & A rabidopsis lyrata subsp. lyrata LEA 1 & & \\
\hline \multirow[t]{2}{*}{ Group 5} & c14252_g1 & 999 & Capsella rubella LEA D-29 & & \\
\hline & c10119_g1 & 618 & A rabidopsis lyrata subsp. lyrata LEA 47 & & \\
\hline \multirow[t]{3}{*}{ Group 6} & c18705_g1 & 886 & A rabidopsis thaliana $L E A D 34$ & & \\
\hline & c5774_g1 & 534 & Camelina sativa LEA 49 & & \\
\hline & c26899_g1 & 183 & A rabidopsis thaliana LEA 3 & & \\
\hline \multirow{5}{*}{ Group 7} & c6277_g1 & 246 & Camelina sativa LEA 5-like & & \\
\hline & c12355_g1 & 510 & A rabidopsis thaliana $L E A 5$ & & \\
\hline & c29783_g1 & 324 & Capsella rubella LEA 41 & & \\
\hline & c13858_g1 & 750 & $\begin{array}{r}\text { Arabidopsis thalianaLEA hydroxyproline rich } \\
\text { glycoprotein family }\end{array}$ & & \\
\hline & c10900_g1 & 552 & A rabidopsis thaliana LEA 27 & & \\
\hline \multirow{4}{*}{$\begin{array}{l}\text { Addition } \\
\text { group } \\
\text { (LEA 2) }\end{array}$} & c13993_g1 & 627 & $\begin{array}{r}\text { Arabidopsis thalianaLEA hydroxyproline rich } \\
\text { glycoprotein family }\end{array}$ & & \\
\hline & c15049_g1 & 702 & $\begin{array}{r}\text { Arabidopsis thalianaLEA hydroxyproline rich } \\
\text { glycoprotein family }\end{array}$ & & \\
\hline & c11524_g1 & 768 & $\begin{array}{r}\text { Arabidopsis thalianaLEA hydroxyproline rich } \\
\text { glycoprotein family }\end{array}$ & & \\
\hline & c17381_g1 & 864 & $\begin{array}{r}\text { Arabidopsis thalianaLEA hydroxyproline rich } \\
\text { glycoprotein family }\end{array}$ & & \\
\hline \multirow{2}{*}{$\begin{array}{l}\text { Addition } \\
\text { group Atm }\end{array}$} & c29526_g1 & 570 & Eutrema salsugineum LEA M 17 & & \\
\hline & c12672_g1 & 375 & Eutrema salsugineum LEA & & \\
\hline
\end{tabular}

Fig. 2. Relative expression analysis of late embryogenesis abundant (LEA) protein family genes before and after germination at a low temperature in Lepidium apetalum seeds based on transcriptome analysis. 
remaining four genes showed no significant difference based on transcriptional data (Fig. 4 Suppl.). The c37384 g1 sequence was selected for further analysis based on the relationship between its expression and abiotic stress. This sequence was named LaLEA1 in this study.

Via cloning and amplification of LaLEAl from L. apetalum by RT-qPCR, one band of the expected size was obtained. Text to sequence analysis showed that the gene contained a complete open reading frame of $624 \mathrm{bp}$ and encoded 208 amino acids (Fig. 5 Suppl.). Bioinformatic analysis showed that the molecular mass of the protein encoded by this gene was 22294.12 . The protein was rich in hydrophilic amino acids. The threonine content was $17.9 \%$, glutamine content was $12.1 \%$, and lysine content was $11.6 \%$, whereas the highest content of hydrophobic amino acids was content alanine $(15.9 \%)$. Thus, the protein was highly hydrophilic. The tertiary structure of the protein was predicted to be dominated by $\alpha$-helices, covering $72.95 \%$ of the peptide chain. Irregular curls accounted for $17.39 \%$ of the peptide (Fig. $6 A$ Suppl.). The secondary structure suggests the protein is folded into an ellipsoid (Fig. $6 B$ Suppl.). Side chains of polar amino acid residues were observed on the surface of the protein; thus, the protein is highly hydrophilic and able to bind water.

$B L A S T$ analysis revealed 10 species with LEA amino acid sequences close to LaLEA1, as identified in GenBank. A phylogenetic tree of the LEA proteins was constructed. LaLEA1 was found in one branch along with LEA76 of Camelina sativa and LEA29 of Capsella rubella, indicating that the conservation of LEA protein was tightly coupled to species (Fig. 3).

Homology analysis of the amino acid sequence of LaLEA1 and the LEA of 10 plant species revealed that the proximal $\mathrm{C}$ - and $\mathrm{N}$-terminal sequences of the proteins were conserved, but the amino acid sequence in the middle (amino acids 30 - 180) varied significantly and the overall homology was relatively low (Fig. 7 Suppl.).

Transcriptome analysis revealed that the LaLEA1 gene was highly expressed during the low-temperature germination. We further investigated whether this gene was involved in the response to short-term low temperature stress. For this purpose, 7-d-old seedlings of L. apetalum were exposed to $0{ }^{\circ} \mathrm{C}$. After $2 \mathrm{~h}$, the expression of LaLEA1 began to increase, reaching its highest level at $8-12 \mathrm{~h}$. Expression began to decrease after $24 \mathrm{~h}$ and, after $48 \mathrm{~h}$ expression was stable but still higher than that prior the treatment, suggesting that the LaLEA1 protein might play a role in the response to low temperature stress.

In contrast to exposure to low temperature, the seedlings under salt stress firstly wilted and then gradually recovered. The wilting rate increased with increased $\mathrm{NaCl}$ concentration. Following treatments with $50-300 \mathrm{mM} \mathrm{NaCl}$, all of the seedlings recovered completely at $48 \mathrm{~h}$ (Fig. $5 A$ ). To investigate whether salt stress influence the expression of LaLEA1, seedlings were treated with 150 and $300 \mathrm{mM} \mathrm{NaCl}$. In the early stage of salt stress, the expression of LaLEA1 increased rapidly (Fig. 5B). Following treatment with $300 \mathrm{mM} \mathrm{NaCl}$ for $6 \mathrm{~h}$, the seedling was highest and the expression of LaLEAI was also the highest, up to 7.9-fold that of untreated seedlings. The rate of seedling wilting was also high at $6 \mathrm{~h}$ following treatment with $150 \mathrm{mM} \mathrm{NaCl}$; however, the highest expression of LaLEA1 was detected at $12 \mathrm{~h}$. These results suggested that salt stress induced LaLEA1 expression, and the expression was positively correlated with the $\mathrm{NaCl}$ concentration. With prolonged salt stress,

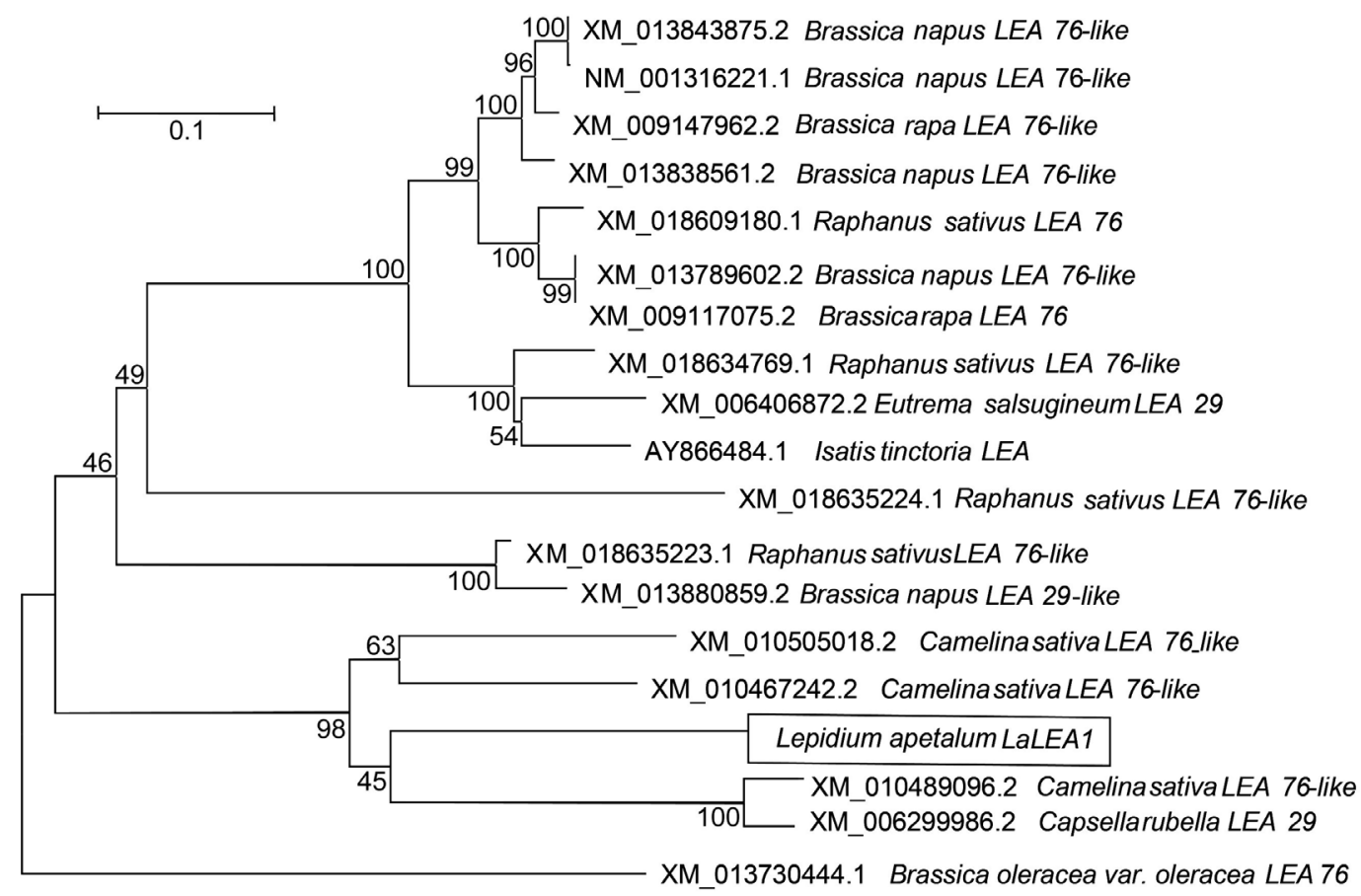

Fig. 3. A phylogenetic tree of Lepidium apetalum late embryogenesis abundant 1 (LaLEA1) with the amino acid sequences of LEA from other species. The numbers at nodes represent the bootstrap values based on 500 replicates. The scale represents genetic distance. 
the expression of LaLEA1 following treatment with both 150 and $300 \mathrm{mM} \mathrm{NaCl}$ decreased. However, a relatively high expression was maintained. These results suggest that LaLEA1 may play an important role in salt tolerance of L. apetalum seedlings.

Drought conditions were simulated by treatment with different concentrations of PEG 6000. Similar to salt

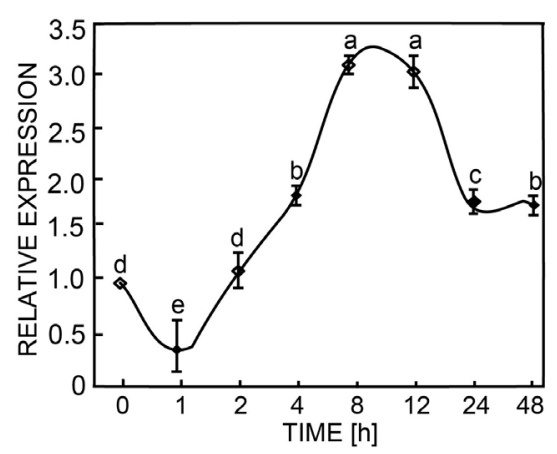

Fig. 4. Lepidium apetalum late embryogenesis abundant 1 (LaLEA1) expression in L. apetalum seedlings in response to short-term low temperature stress $\left(0^{\circ} \mathrm{C}\right)$. Means \pm SEs, $n=3$. Different letters indicate significant differences $(P<0.05$; the Student's t-test).

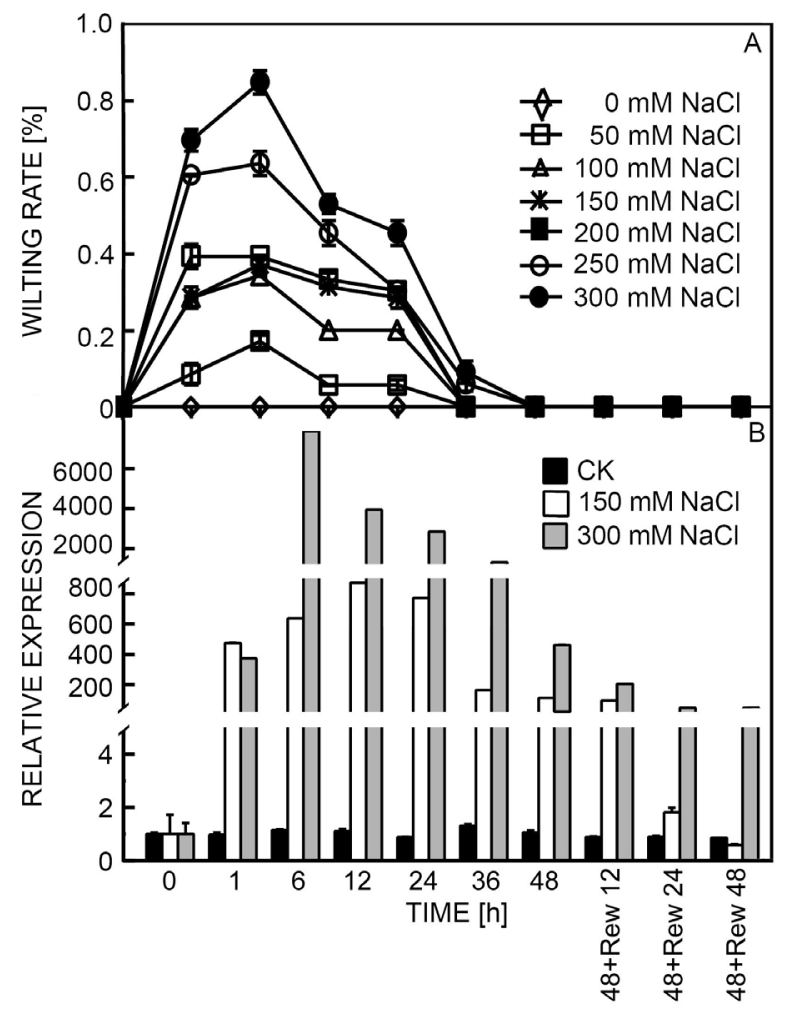

Fig. 5. Wilting rate of Lepidium apetalum seedlings. $A$ - Wilting the seedlings treated with different concentrations of $\mathrm{NaCl}$ and different stress duration. Slightly yellow and curly leaves were thought to have wilted. $B$ - Quantitative fluorescence of L. apetalum late embryogenesis abundant 1 (LaLEA1) expression in L. apetalum seedlings treated with different concentrations of $\mathrm{NaCl}$ for diferrent duration (48+Rew is seedling recovery at $0 \mathrm{mM} \mathrm{NaCl}$ for 12,24 , and $48 \mathrm{~h}$ after 48 -h salt stress). stress, drought treatment can cause temporary wilting and with prolongation of the processing time, wilting was restored. However, unlike salt stress, the wilting was not reversible in all cases; high concentrations of PEG 6000 can cause permanent wilting. Wilting was completely reversed within $48 \mathrm{~h}$ with lower concentrations (10 and $15 \%$ of PEG 6000 ), whereas it was only partially reversed at 25 and $30 \%$ PEG 6000. Treatment of seedlings with $20 \%$ PEG 6000 caused wilting that was almost completely reversible. Therefore, seedlings treated with 20 and $30 \%$ PEG 6000 were chosen to investigate the relationship between LaLEA1 expression and osmotic stress. Our results showed that, with an increase in the wilting rate, LaLEA1 expression rapidly increased in the early stage of stress. With the prolongation of osmotic stress, the expression of LaLEA1 decreased gradually. However, a higher expression was maintained compared to that in control. Following treatment with $30 \%$ PEG 6000, the wilting rate and LaLEA1 expression were highest at $24 \mathrm{~h}$; the expression of LaLEA1 was up to 6.5-fold higher than that in control. Following treatment with $20 \%$ PEG 6000 , the highest LaLEA1 expression occurred at $12 \mathrm{~h}$, whereas the highest wilting rate was observed at $24 \mathrm{~h}$. These results suggest that the increase in $L a L E A 1$ expression was related to the severity of the osmotic stress and that the LaLEAI gene may play an important role in drought tolerance of L. apetalum seedlings. These results are similar to those obtained under low temperature and salt stress.

\section{Discussion}

The number of LEA family genes differs among plants. It has been reported that there are 36 LEA genes in Glycine max (Li et al. 2011), 27 in Ricinus communis (Zou et al. 2013), 23 in Medicago truncatula (Liu et al. 2015), 29 in Solanum tuberosum (Safa et al. 2015), 27 in Solanum lycopersicum (Cao and Li 2015), and 87 in Poplar sp. (Li et al. 2016). Here, 27 genes encoding LEA family proteins were identified from the transcriptome of $L$. apetalum seeds during germination at low-temperature. It is possible that some LEA protein coding genes were not expressed in the germinated seeds of L. apetalum, and thus the number of LEA protein family genes in this plant may be greater than 27. More experimental evidence is needed to determine the number of LEA protein family coding genes in L. apetalum.

As an early spring ephemeral plant, the germination of L. apetalum seeds is an interesting process. Based on the transcriptome data, expression of the LaLEA1 gene was high in L. apetalum seeds, which were able to germinate at low temperature, as well as being higher than that of seeds that could not germinate significantly at low temperature. It has been suggested that the LaLEA1 gene plays an important role in the tolerance of seed germination to low temperature. It has also been shown that the amino acid sequence of the protein encoded by LaLEA1 was highly homologous to that of LEA76, of Camelina sativa and Brassica campestris. In terms of structure classification, LaLEA1 and LEA76 belong to the third group of LEA 
proteins. Members of this group of LEA proteins have been reported to bind to hydrophobic surfaces to form dimers, thereby performing an ionic chelating function, when plants are dehydrated. Dure and Galau (1976) reported that LEA76 has 13 repetitive motives, but did not specify the function of LEA76 for plants in KOG

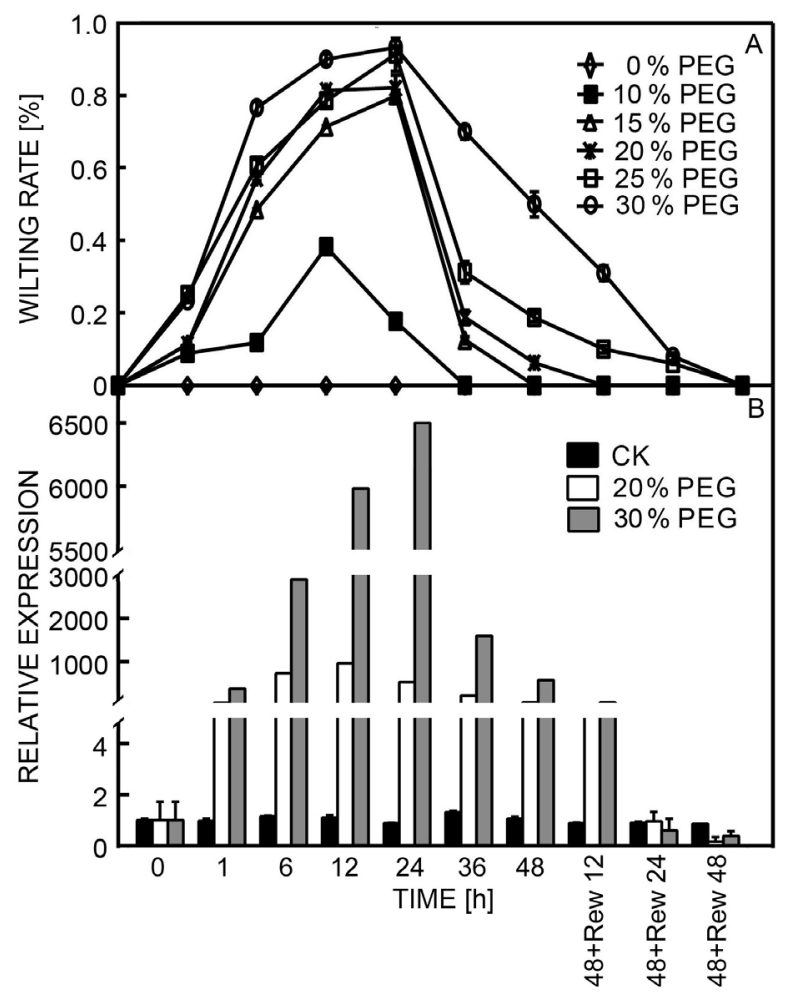

Fig. 6. Wilting rate of Lepidium apetalum seedlings. A - Wilting the seedlings treated with different concentrations of PEG 6000 for different stress duration. Slightly yellow and curly leaves were thought to have wilted. $B$ - Quantitative fluorescenceanalysis of L. apetalum late embryogenesis abundant 1 (LaLEA1) expression in L. apetalum seedlings treated with PEG 6000 for different duration $(48+\mathrm{Rew}$ is the seedlings recovery at $0 \mathrm{mM} \mathrm{NaCl}$ for 12,24 , and $48 \mathrm{~h}$ after 48 -h salt stress). annotations. The secondary structure of the LaLEA1 protein is primarily composed of $\alpha$-helices, and dimers are formed by hydrophobic interactions. The side chains of hydrophilic amino acids are distributed on the surface of the molecule, rendering the protein more bound water. Because of its hydrophilic properties, LaLEA1 can bind several water molecules. When plant cells are exposed to low temperature stress, the LEA proteins enhance plant tolerance to that stress by lowering the freezing point of bound water. Under salt and osmotic stress, the hydrophilic LEA proteins maintain cell water content via their strong water binding capacity, reducing the amount of damage caused by loss of water. This result is consistent with the characteristics of L. apetalum seedlings, which are tolerant to low temperature, high salinity, and drought. We believe that the expression of LaLEA1 is closely related to plant tolerance to abiotic stresses, such as low temperature, salinity, and drought.

The RT qPCR indicated that the expression of LaLEA1 was sensitive to low temperature stress, such as $0^{\circ} \mathrm{C}$. In the early stage of stress (within $1 \mathrm{~h}$ ), the expression decreased significantly, and then increased rapidly; expression was highest at $8-12 \mathrm{~h}$. This suggests that the change in gene expression is not a direct response to low temperature stress, but rather to a later step in signal transduction (Fig. 7). One possible explanation is that the expression of LaLEA1 at the early stage of cold stress increased sharply in responce to sudden hypothermia. Over time, the seedlings gradually adapted to the low temperature; while the expression of LaLEA1 decreased slowly, it still remained at a relatively high level. This latter phenomenon suggests that LaLEA1 may play a role in the regulation of seedling growth under sustained low temperature.

Following salt or osmotic stress, L. apetalum seedlings wilt rapidly and the greater the $\mathrm{NaCl}$ or PEG concentration the higher the rate of wilting. However, when the stress was present for more than $48 \mathrm{~h}$, most of the seedlings returned to normal, i.e., the wilting rate decreased significantly. Interestingly, the change in LaLEA1 gene expression was closely linked to the change in seedling wilting rate. That

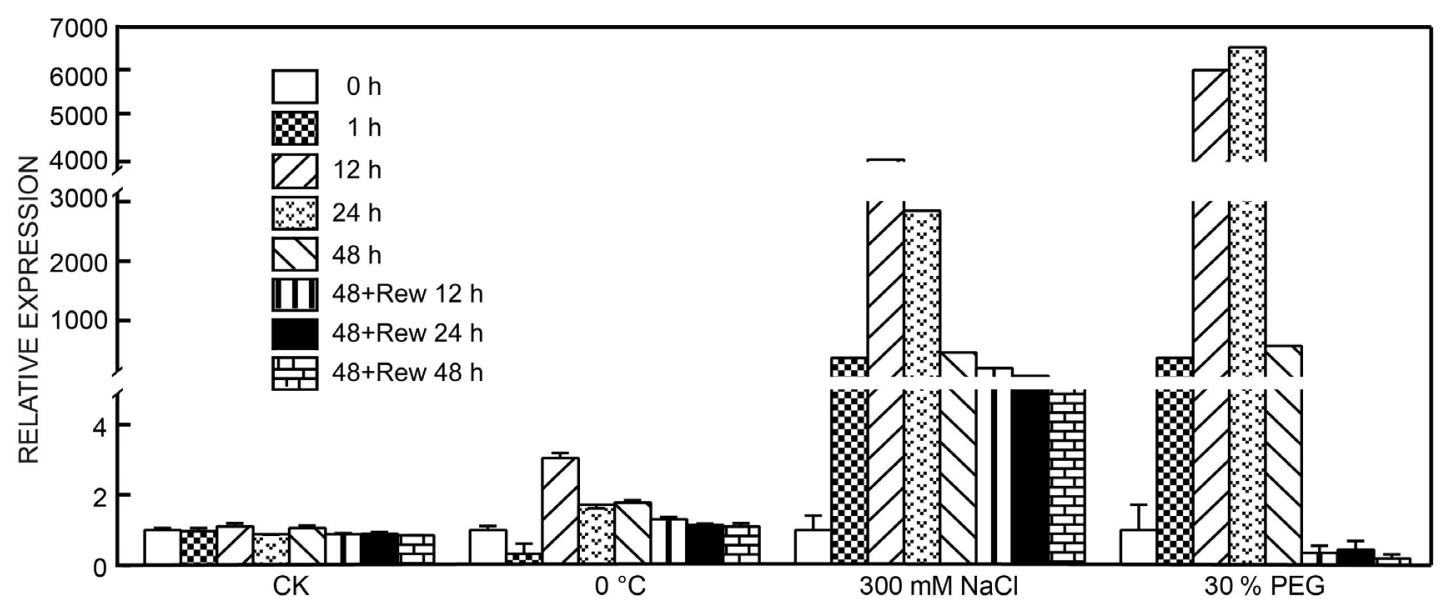

Fig. 7. Lepidium apetalum late embryogenesis abundant 1 (LaLEA1) expression in L. apetalum seedlings in response to abiotic stresses for $0,1,12,24$, and $48 \mathrm{~h}, \mathrm{CK}$ - 7-d-old control seedling, 48+Rew - seedlings recovered at $0 \mathrm{mM} \mathrm{NaCl}$ for 12, 24, and $48 \mathrm{~h}$ after 48 -h salt stress. 
is, seedlings wilted rapidly when under both drought or salt stresses, and the expression of LaLEA1 also increased rapidly in a short time; the wilting rate and expression of LaLEA1 was highest at similar times. Then, the seedlings gradually returned to normal; expression of LaLEA1 decreased but remained higher than that under non-stress conditions.

These results suggest that LaLEA1 expression might play an important role in the tolerance of $L$. apetalum seedlings to low temperature, salinity, and osmotic stress although additional experimental evidence is needed to determine whether LaLEA1 increases the ability of plants to retain water.

\section{References}

Alan, T., Wise, M.J.: The continuing conundrum of the LEA proteins. - Naturwissenschaften 94: 791-812, 2007.

Arroyo, A.G., Colmenero-Flores, J.M., Garciarrubio, A.: Highly hydrophilic proteins in Prokaryotes and Eukaryotes are common during conditions of water deficit. - J. biol. Chem. 275: 5668-5674, 2000.

Cai, D., Zheng, Y.Z., Lan, Y.: The expression of soybean LEA protein Em can improve salt tolerance of Escherichia coli and tobacco. - J. Shenzhen Univ. 23: 230-236, 2006.

Cao, J., Li, X.: Identification and phylogenetic analysis of late embryogenesis abundant proteins family in tomato (Solanum lycopersicum). - Planta 241: 757-772, 2015.

Capdevila, A.M., Dure, L.: Developmental biochemistry of cottonseed embryogenesis and germination. - Plant Physiol. 68: 187-194, 1981

Dure, L., Galau, G.A.: Developmental biochemistry of cottonseed embryogenesis and germination. XIII. Regulation of biosynthesis of principal storage proteins. - Plant Physiol. 101: 503-536, 1976.

Galau, G.A., Bijaisoradat, N., Hughes, D.W.: Accumulation kinetics of cotton late embryogenesis-abundant mRNAs and storage protein mRNAs: coordinate regulation during embryogenesis and the role of abscisic acid. - Dev. Biol. 123: 198-212, 1987

He, D.Y., Xia, R.J., Zhou, L.J., Xu, P.L.: Improvement of drought and salt tolerance in tobacco by over expression of $L E A 5$ gene in soybean. - Acta Laser Biol. sin. 27: 53-59, 2018.

He, S., Hu, Y.Z.: Protective mechanism of OsLEA5 protein on lactate dehydrogenase in vitro. - Model. Med. Health 30: 1445-1447, 2015.

Hu, Y.Z., Chen, Z.G., Juan, Q., Huang, X.Y.: Expression, purification and protective effect of OsLEA5c in Escherichia coli. - J. Henan agr. Sci. 44: 11-14, 2015.

Ingram, J., Bartels, D.: The molecular basis of dehydration tolerance in plants. - Annu. Rev. Plant Physiol. Plant mol. Biol. 47: 377-??, 1996.

Jiang, Q.Y., Li, L.L., Niu, F.J., Sun, X.J., Hu, Z., Zhang, H.: Overexpression of TaLEA1 and TaLEA2 genes enhances salt tolerance in transgenic Arabidopsis. - J. Plant Genet. Resour. 18: 509-519, 2017.

Li, C.N., Gao, Y., Liu, Z.M., Liu, G.B., Zheng, Y.Z.: Antioxidant activity of soybean PM1 protein and improvement of copper tolerance of yeast. - J. Shenzhen Univ. 34: 457-463, 2017a.

Li, L., Xu, H.L., Yang, X.L., Li, Y.X., Hu, Y.K.: Genome-wide identification, classification and expression of LEA gene family in soybean. - Agr. Sci. China 44: 3945-3954, 2011.

Li, L.L., Zeng, W.J., Li, Y.H., Ge, F.W., Lu, H., Lei, W., Du,
Y., Xie, H.T., Zhao, H.P., Zhao, H.X.: Analysis of GRAS transcription factor family and cloning of LaSCL18 gene in Lepidopsis uniquely and its cold-related study. - Mol. Plant Breed. 15: 3428-3437, $2017 \mathrm{~b}$.

Li, J., Zhao, A,C., Liu, C,Y., Wang, Q,L., Liu, X,Q., Yu, M,D.:Whole-genome identification and expression analysis of poplar LEA gene family. - J. Southwest. Univ. 8: 48-56, 2016.

Li, P.P., Zeng, W.J., Zhou, Q., Zhao, H.X., Li, Y.H., Ge, F.W., Zhu, C.Q., Zhao, J.J., Lu, H., Zhao, H.P.: Screening and expression analysis of temperature response proteins to remove the stagnation of low temperature germination of Lepidopsis uniflora seeds. - Mol. Plant Breed. 14: 722-729, 2016.

Liang, Y.J., Yu, Z.Y., Qiang, Z.Q., Du, Y., Zhu, W.N., Zhang, D.P., Zhang, L.S.: Cloning and stress induced expression analysis of TaDRLea 3-2 gene in wheat. - Acta bot. borealioccidentalia sin. 36: 1091-1097, 2016.

Lin, H., Zeng, X., Guo, S.X.: Cloning, expression and functional analysis of DoLEA2 gene from Dendrobium candidum. - Acta pharmacol. sin. 52: 139-146, 2017.

Liu, G., Xu, H., Zhang, L., Zheng, Y.: Fe binding properties of two soybean (Glycine max L.) LEA 4 proteins associated with antioxidant activity. - Plant Cell Physiol. 52: 994-1002, 2011.

Liu, Y., Wang, L., Jiang, S.S., Pan, J.W., Cai, G.H., Li, D.Q.: Group 5 LEA protein, Zmlea5c, enhance tolerance to osmotic and low temperature stresses in transgenic tobacco and yeast. - Plant Physiol. Biochem. 8: 22-31, 2014.

Liu, Z.M., Liu, W.X., Liu, X.T., Zhang, Z.S., Wang, Y.R.: Genome-wide analysis of $L E A$ gene family in alfalfa tribulus. - Pratacult. Sci. 32: 382-391, 2015.

Mao, Z.M., Zhang, X.M.: Flora outline of early spring short-lived plants in northern Xinjiang. - Arid Zone Res. 11: 1-26, 1994.

Meng, J., Li, Q., Li, G.: Study on the physiological characteristics of seed germination of two species of Lepidopsis. Biotechnology 18: 32-35, 2008.

Mouillon, P., Gustafsson, P., Harryson, P.: Structural investigation of disordered stress proteins. comparison of full-length dehydrins with isolated peptides of their conserved segments. - Plant Physiol. 141: 638-650, 2006.

Oliveira, E., Amara, I., Bellido, D., Odena, M.A., Domínguez, E., Pagès, M., Goday, A.: LC-MSMS identification of Arabidopsis thaliana heat-stable seed proteins: enriching for LEA-type proteins by acid treatment. - J. Mass Spectrometry 42: 1485-1495, 2010.

Safa, C., Najib, S.d.M., Mariam, C., Radhia, G.B.: Genomewide identification and expression profiling of the late embryogenesis abundant genes in potato with emphasis on dehydrins. - Mol. Biol. Rep. 42: 1163-1174, 2015.

Shah, S., Weinholdt, C., Jedrusik, N., Molina, C., Zou, J., Große, I., Schiessl, S., Jung, C., Emrani, N.: Whole-transcriptome analysis reveals genetic factors underlying flowering time regulation in rapeseed (Brassica napus L.). - Plant Cell Environ. 41: 1935, 2018.

Wang, B.F., Wang, Y.C., Zhang, D.W., Li, H.Y., Yang, C.P.: The expression of LEA gene in Saccharomyces cerevisiae enhances the resistance of yeast to abiotic stresses. - J. Forest. Res. 19: 58-62, 2008.

Wang, S., Liu, M.R., Huang, H.J., Mu, H.Z., Li, Z.X., Liu, G.F.: Screening of cold-resistant lines of TaLEA transgenic Populus nigra. - J. Forest Res. 39: 5-7, 2011.

Xiang, D.J., Man, L.L., Zhang, C.L., Peng, L., Li, Z.G., Zheng, G.C.: A new Em-like protein from Lactuca sativa. LsEm1 enhances drought and salt stress tolerance in Escherichia coli and rice. - Protoplasma 255: 1-18, 2018.

Yang, N., Zhao, H.P., Ge, F.W., Li, Y.H., Zeng, W.J., Zhao, H.X.: Physiological response of germination of two species 
of Lonicerae to low temperature stress. - Arid Zone Res. 32: 760-765, 2015.

Young, B.M., Hua, C.L., Jungeun, L., Hyun, P., Andosung, L., Taek, K.W., Hyoungseok, L.: Identification of rice genes associated with enhanced cold tolerance by comparative transcriptome analysis with two transgenic rice plants overexpressing dacbf4 or dacbf7, isolated from antarctic flowering plant Deschampsia antarctica. - Front. Plant Sci. 9: 601-10, 2018

Yu, J., Lai, Y., Wu, X., Wu, G., Guo, C.: Overexpression of OsEml encoding a group I LEA protein confers enhanced drought tolerance in rice. - Biochem. biophys. Res. Commun. 478: 703-709, 2016.

Yu, X.M., Yue, W.R., Zhang, Y.N., Wang, R.G., Jing, L.G., Yang, Q.: Cloning and functional analysis of CkLEA4-2 gene from Caragana korshinskii. - Acta pPhytophysiol. sin. 10: 94-105, 2017.

Yuan, L.L., Wang, Y.R., Zeng, W.J., Xie, H.T., Du, Y., Lu, H., Zhao, H.X.: Response of $b H L H$ transcription factor gene family and laICE1 expression in seedlings of uninhabited vegetable to cold stress. - Acta bot. boreali-occidentalia sin. 38: 0026-0034, 2018.

Zhang, H., Liu, Y.Y., Chen, Y., Ping, S.Z., Wang, J.: Biological function of Dgl5 protein of abnormal Gobi coccus. Biotechnol. Bull. 34: 177-184, 2018.

Zhao, H.X., Li, Q., Jin, Z.: Tolerance to low temperature during seed germination of short-lived plants. - Plant Diversity Resour. 32: 448-454, 2010.

Zhao, H.X., Zhao, Q.Q., Lu, H., Zhu, Y.L., Yu, L.J., Qian, H.B., Du, Y., Xie, H.T., Zhao, W.J., Zeng, W.J.: Response of DREB transcription factor family and $L a D R E B$ expression to cold stress in seedlings of Lepidopsis uniquely. - Mol. Plant Breed. 16: 42-48, 2018.

Zhou, Q., Zhao, H.X., Li, P.P., Zeng, W.J., Li, Y.H., Ge, F.W., Zhao, J.J., Zhao, H.P.: High-throughput sequencing and analysis of the transcriptome of the seeds of Lepidopsis uniflora. - China Biotechnol. 36: 38-46, 2016.

Zou, Z., Huang, Q.X., An, F.: Genome-wide identification, classification and evolution analysis of LEA gene family in castor. - Chin. J. Oil Crop Sci. 35: 637-643, 2013. 